\title{
Mesoscopic simulations of systolic flow in the human abdominal aorta ${ }^{\star 2}$
}

\author{
A.M. Artoli*, A.G. Hoekstra, P.M.A. Sloot \\ Section Computational Science, Laboratory for Computing, Systems Architecture and Programming, Faculty of Science, University of Amsterdam, \\ Kruislaan 403, 1098 SJ Amsterdam, The Netherlands
}

Accepted 26 January 2005

\begin{abstract}
The complex nature of blood flow in the human arterial system is still gaining more attention, as it has become clear that cardiovascular diseases localize in regions of complex geometry and complex flow fields. In this article, we demonstrate that the lattice Boltzmann method can serve as a mesoscopic computational hemodynamic solver. We argue that it may have benefits over the traditional Navier-Stokes techniques. The accuracy of the method is tested by studying time-dependent systolic flow in a 3D straight rigid tube at typical hemodynamic Reynolds and Womersley numbers as an unsteady flow benchmark. Simulation results of steady and unsteady flow in a model of the human aortic bifurcation reconstructed from magnetic resonance angiography, are presented as a typical hemodynamic application.
\end{abstract}

(C) 2005 Elsevier Ltd. All rights reserved.

Keywords: Lattice Boltzmann method; Abdominal aorta; Systolic flow; Cardiovascular diseases

\section{Introduction}

Flow characteristics near branches and bifurcations are quite important in hemodynamics: Cardiovascular diseases are considered as a leading cause of death in the developed world and are now becoming more prevalent in developing countries (World Health Organization, 2002). Most of these diseases localize in regions of complex geometry of the arterial tree. The flow fields and shear stress play important roles in understanding, diagnosis and treatment of such diseases. Although being studied by many authors (e.g. Womersley, 1955; McDonald, 1974; Caro et al., 1978; Ku et al., 1985; Moore et al., 1994a, b; Moore and Ku, 1994a; Reneman

\footnotetext{
${ }^{2}$ Supported in part by the Soedanees Dutch Foundation, Leiden, The Netherlands.

*Corresponding author. Tel.: + 31205257462 ; fax: +3120525 7490.

E-mail addresses: artoli@science.uva.nl (A.M. Artoli), alfons@ science.uva.nl (A.G. Hoekstra), sloot@science.uva.nl (P.M.A. Sloot).

URL: http://www.science.uva.nl/research/scs/.
}

et al., 1993; Taylor et al., 1996; Gijsen et al., 1997; Vorp et al., 1998; Wootton and $\mathrm{Ku}, 1999)$, the relation between flow fields and cardiovascular diseases is still not fully understood, and is currently receiving more and more attention (Botnar et al., 2000; Berthier et al., 2002; Cheng et al., 2002).

There have been many reports relating low and oscillatory shear stress to atherosclerosis in large arteries. For a recent review, we refer to Shaaban and Duerinckx (2000). Frequently, the treatment of an arterial disease involves implanting a new host artery as an additional or a replacement to the diseased one, or design of a cardiovascular device. These are quite difficult to plan and have to be tailor made for each specific patient.

Recently, two major developments in the field of vascular surgery planning have made it possible to better and faster plan risk reduced implantation: Firstly, magnetic resonance imaging angiography (MRA) has been significantly enhanced to provide excellent images of the arterial tree and non-invasive dynamic data 
acquisition is made possible (Goyen et al., 2001). Secondly, the development of cheap computing power and interactive simulation environments have made near real time simulations come within reach (Taylor et al., 1999; Belleman and Sloot, 2000; Zhao et al., 2002). With these in hand, an efficient and robust flow solver can be used as an interactive modeling environment. There are various successful computational fluid dynamics (CFD) methods commonly used here, such as the finite-element methods, the finite difference methods and the finite volume methods. All these techniques are well established, but they yield three major difficulties (Taylor et al., 1998): the non-trivial and time consuming grid generation, the necessity to solve the Poisson equation for the pressure field, and the approximations involved in computing the shear stress from computed velocity fields.

On the other hand, the conventional flow solvers such as the finite-element methods and the finite difference methods are accurate and efficient. However, their applicability to problems involving complex and moving geometry is complicated, due to their strong dependence on time consuming mesh generation. The Navier-Stokes (NS) equations can now be solved with mesh-free algorithms (Batina, 1993) which are unfortunately not flexible enough due to errors attributed to numerical viscosity and to difficulty in improving the space and time accuracy. Dynamic mesh generating techniques have been reported recently (Taylor et al., 1998).

New particle-based methods such as dissipative particle dynamics, lattice gases and lattice Boltzmann methods have been developed and matured (Mcnamara and Zanetti, 1988; Higuera and Succi, 1989; Qian et al., 1992; Aharonov and Rothman, 1993; Behrend, 1995). These mesoscopic techniques may be quite useful for hemodynamic research, as, among other features, they are more flexible in dealing with multicomponent fluid flow problems, require simple regular meshes, and are less sensitive to computational complexity in complicated geometry. In this study, we use the lattice Boltzmann BGK method, shortly described in the next section.

Since the shear rate in the aorta is higher than $0.1 \mathrm{~s}^{-1}$, we consider blood to be Newtonian. We also ignore the elastic behavior of the aortic walls for its minor effects on the flow fields in the aorta. It is well known that due to the pulsatile behavior of blood flow and the elasticity of the arteries, the diameter of the larger arteries may vary $5-10 \%$ during a systolic cycle (e.g. Taylor et al., 1998). However, in typical hemodynamic simulations the computational mesh is obtained from magnetic resonance imaging of real arteries. Such images typically have errors in the position of the artery lumen of $1-8 \%$ (Moore et al., 1998). Therefore, given this accuracy, the influence of elasticity of the wall can be considered a secondary effect, which we did not include in our simulations. However, we have been working on elastic walls in our simulations, and first results, validating lattice Boltzmann BGK method with including wall elasticity were obtained (Hoekstra et al., 2004).

\section{Numerical method}

The numerical method used in this study is based on the lattice Boltzmann method, a newly adapted mesoscopic Eulerian solver. It is a discretization of the Boltzmann equation that describes the evolution of particles in kinetic theory. Due to its simple implementation, straightforward parallelization and easy grid generation, the capability of the lattice Boltzmann method has been demonstrated in various complex applications including Newtonian blood flow simulations (Krafczyk et al., 1998; Artoli et al., 2002), nonNewtonian and suspension flows (e.g. Ladd, 1994), and complex geometry (e.g. Kandhai et al., 1999). As timedependent flow simulations are known to be computationally expensive, a need for an efficient flow solver is crucial. Traditional NS solvers frequently use artificial compressibility and pressure projection methods to accelerate convergence. In this study, we present the capability of the lattice Boltzmann BGK method as a robust technique for systolic Newtonian flow in a 3D rigid tube and in a complex model of the human abdominal aorta reconstructed from MRA images of a volunteer.

Different from the traditional CFD methods which obtain the velocity and pressure by solving the NS equations and compute the shear stress from the velocity profiles, the lattice Boltzmann BGK method utilizes a special finite difference equation of the simplified Boltzmann BGK equation which describes transport phenomena at the mesoscopic level. It is useful to shortly introduce the method and highlight its capabilities to encourage researchers in hemodynamics to consider it as an alternative approach for complex flow problems. Detailed descriptions of the method and demonstrations of its capability have been presented in literature as cited above.

The dynamics of the fluid is modeled by the transport of simple fictitious particles on the nodes of a Cartesian grid and is based on two steps; streaming to the neighboring nodes and colliding with local node populations represented by the probability $f_{i}$ of a particle moving with a velocity $\mathbf{e}_{i}$ per unit time-step $\delta t$. Populations are relaxed towards their equilibrium states during a collision process. The equilibrium distribution function

$f_{i}^{(0)}=w_{i} \rho\left(1+\frac{3}{v^{2}} \mathbf{e}_{i} \cdot \mathbf{u}+\frac{9}{2 v^{4}}\left(\mathbf{e}_{i} \cdot \mathbf{u}\right)^{2}-\frac{3}{2 v^{2}} \mathbf{u} \cdot \mathbf{u}\right)$ 
is a low Mach number approximation to the expansion of the Maxwellian distribution. Here, $w_{i}$ is a weighting factor, $v=\delta x / \delta t$ is the lattice speed, and $\delta x$ and $\delta t$ are the lattice spacing and the time-step, respectively. The values of the weighting factor and the discrete velocities depend on the used lattice Boltzmann model and can be found in literature (e.g. Chopard and Droz, 1998; Succi, 2001). The lattice Boltzmann BGK equation

$f_{i}\left(\mathbf{x}+\mathbf{e}_{i} \delta t, \mathbf{e}_{i}, t+\delta t\right)-f_{i}\left(\mathbf{x}, \mathbf{e}_{i}, t\right)=\Omega$

can be obtained by discretizing the evolution equation of the distribution functions in the velocity space using a finite set of velocities $\mathbf{e}_{i}$. In this equation, $\Omega=$ $-(1 / \tau)\left[f_{i}\left(\mathbf{x}, \mathbf{e}_{i}, t\right)-f_{i}^{(0)}\left(\mathbf{x}, \mathbf{e}_{i}, t\right)\right]$ is the so-called BGK collision term and $\tau$ is a dimensionless relaxation time. The hydrodynamic density, $\rho$, and the macroscopic velocity, $\mathbf{u}$, are determined in terms of particle distribution functions using the laws of conservation of mass and momentum:

$$
\begin{aligned}
& \rho=\sum_{i} f_{i}=\sum_{i} f_{i}^{(\mathrm{eq})} \quad \text { and } \\
& \rho \mathbf{u}=\sum_{i} \mathbf{e}_{i} f_{i}=\sum_{i} \mathbf{e}_{i} f_{i}^{(\mathrm{eq})} .
\end{aligned}
$$

By Taylor expansion of the lattice Boltzmann BGK equation up to $O\left(\delta t^{2}\right)$ and application of the multi-scale Chapman-Enskog technique through expansion of $f_{i}$ about $f_{i}^{(0)}$, we can derive the NS equations and the momentum flux tensor up to second order in the Knudsen number. The pressure is simply given by $p=$ $\rho c_{\mathrm{s}}^{2}$ and the kinematic viscosity is $v=c_{\mathrm{s}}^{2} \delta t\left(\tau-\frac{1}{2}\right)$, where $c_{\mathrm{s}}$ is the speed of sound. Different lattice BGK models differ in the choice of the distribution functions, the number of moving particles and the speed of sound inside the system. In our study, we use the threedimensional 19 particles (D3Q19) model which has a rest particle, six particles along the principal directions and 12 particles along the diagonals.

Within the lattice Boltzmann community, it has been acknowledged that solving the lattice Boltzmann equation has three main advantages over solving the NS equations: First, it works with fast and easy to generate Cartesian grids while still yield accurate results of second order in space and time. Secondly, the pressure is simply a linear function in the speed of sound $\left(p=\rho c_{\mathrm{s}}^{2}\right)$ while the NS solvers need to solve the Poisson equation. Finally and most important for the field of hemodynamics, is that the stress tensor can be directly obtained from the non-equilibrium parts of the distribution functions $f_{i}^{(1)}$ through the following relation (Ladd, 1994; Artoli et al., 2002)

$\sigma_{\alpha \beta}=-\rho c_{\mathrm{s}}^{2} \delta_{\alpha \beta}-\left(1-\frac{1}{2 \tau}\right) \sum_{i=0} f_{i}^{(1)} e_{i \alpha} e_{i \beta}$

which gives $\alpha \beta$-component of the stress tensor. Here, $\delta_{\alpha \beta}$ denotes the unit tensor, $e_{i \alpha}$ and $e_{i \beta}$ are the relevant lattice speeds. Eq. (3) gives the stress tensor independent of the velocity fields, whereas a need to get the derivative of obtained velocity profiles is not avoidable when NS solvers are used. The non-equilibrium parts of the distribution functions are computed during collision steps to relax the system towards equilibrium. Therefore, Eq. (3) does not involve considerable computational cost. All these advantages make the lattice Boltzmann method a promising candidate for simulating time-dependent blood flow in arteries.

The lattice Boltzmann method can easily be adapted to simulate time-dependent flows such as the flow driven by a cardiac pressure cycle in a tube (Artoli et al., 2002). Since it is a linear function in the pressure, timedependent density gradients can easily be implemented to represent a systolic flow rate. A range of values of the Womersley parameter can be simulated without affecting the stability of the model. All kinds of inlet and outlet boundary conditions, usually applied in computational hemodynamics, can equivalently be implemented. The robustness of the method appears in the straightforward parallelization of the easy to generate Cartesian grid. On the other hand, since it is implemented on a Cartesian grid, very fine grids need to be generated to simulate flow in complex geometry. Filippova and Hänel (1998) have overcome this problem by considering local grid refinement.

\section{Simulations}

\subsection{Systolic flow in a tube}

The aim of this benchmark is to investigate the accuracy of the lattice Boltzmann BGK method in modeling applications related to hemodynamics and biomedical Engineering.

We have conducted a number of $3 \mathrm{D}$ simulations of a systolic flow in a rigid tube. The diameter of the tube $D$ is represented by 40 lattice nodes and the tube length is $L=80$ lattice nodes. Oscillatory components of the pressure gradient derived from the pressure wave (shown at the bottom of Fig. 1) are used to drive the flow. The Reynolds number is defined in terms of the maximum velocity $U$ as $R_{\mathrm{e}}=U D / v$ and the Womersley number is defined as $\alpha=n R \sqrt{\omega / v}$, where $\omega=2 \pi / T$ is the angular frequency, $T$ being the time for one complete heart pulse (the period).

Simulation parameters are tuned to have a Womersley number equal to 16 and an average Reynolds number of 590 , typical values in the abdominal aorta during resting conditions (Moore and $\mathrm{Ku}, 1994 \mathrm{a}$ ). Simulations were performed on 4 nodes of a parallel computer (a Beowulf cluster containing $750 \mathrm{MHz}$ AMD Athlon nodes with 512 MB RAM memory). The time per iteration is about $0.2 \mathrm{~s}$ and the total simulation time is $24 \mathrm{~min}$. 

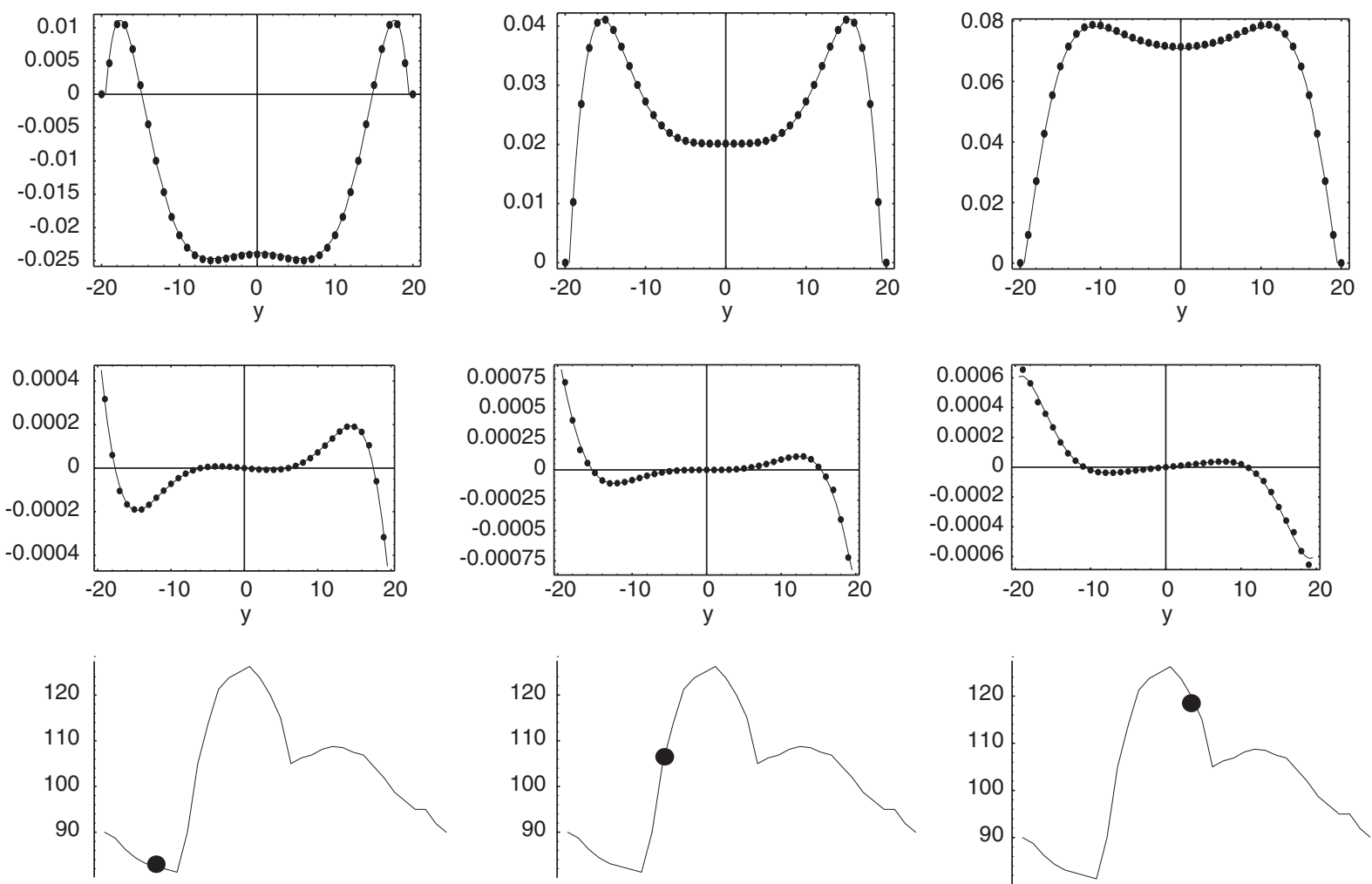

Fig. 1. Oscillatory components of velocity profiles (upper row) and shear stress (middle row) in lattice units at different incidents of the cardiac cycle (bottom): near the end of the isovolumetric contraction (left column), during rapid ejection (middle) and reduced ejection (right) for the 3D tube benchmark. The Reynolds number is 590 and the Womersley parameter is 16 . The dots are simulation results and the solid lines are the Womersley solutions.

The data show excellent agreement with the analytical solutions for the velocity and the shear stress throughout the whole cardiac cycle. Fig. 1 shows some examples of the obtained oscillatory components of velocity and shear stress profiles near the end of the isovolumetric contraction and during rapid ejection. Similar agreement has been observed throughout the cycle. The maximum error in velocity and shear stress is less than $1 \%$ and can even be reduced if the grid is refined and the Mach number is reduced. However, for engineering purposes, this error is quite acceptable, since the anatomy of the arteries already has a comparable uncertainty in the wall positions (Moore et al., 1998). All values are presented in lattice units. To convert to physical dimensions, the time-step and the lattice spacing have to be computed for a given geometry and the viscosity of the blood. The velocity has to be multiplied by $\delta x / \delta t$ and the stress has to be converted to the used pressure units.

We have conducted several experiments in a range of Womersley and Reynolds numbers commonly observed in the human carotid, coronary arteries and arterioles, and obtained similar agreements as those shown in Fig. 1. Therefore, the lattice Boltzmann method is accurate enough to reproduce the analytical Womersley solution.
This motivates us towards having a more realistic model from the arterial tree.

\subsection{Flow in the abdominal aorta}

Atherosclerosis mainly occurs in focused locations of large and medium arteries such as the carotid bifurcation, the coronary arteries, the abdominal aorta and the iliac and femoral arteries at regions of low and oscillating shear stress, independent of other risk factors (Shaaban and Duerinckx, 2000). In the human abdominal aorta, the lateral and posterior walls of the aorta distal to the inferior mesentric artery (IMA) are highly susceptible to atherosclerosis. These regions are known to have low and oscillating shear stress (Gibson et al., 1993; Oshinski et al., 1995; Taylor et al., 1998).

From the arguments raised above and the conducted benchmark experiments, we are further encouraged to explore the capability of the lattice Boltzmann method in simulating a more realistic geometry of interest to Biomechanics. We choose to study flow in a model of the human abdominal aorta as an example. The model is reconstructed from magnetic resonance angiography of a volunteer. The pressure gradient at the entrance of the aorta is averaged from flow rate obtained from literature 
(Moore et al., 1994a; Taylor et al., 1999). The computational model under study involves only the bifurcation region, directly after the IMA, and includes parts of the left and right iliac arteries (see Fig. 2). The complete model of the abdominal aorta, including the celiac, mesenteric and renal branches, and the femoral artery, is currently under study.

Many studies on the flow in the abdominal aorta have been reported, all relating the cause of the disease mainly to the complex nature of the shear stress profiles in these regions. Moore et al. (1994a) computed the shear stress in a model of the human abdominal aorta under resting and exercise conditions from MRA measured velocity data and extracted six shear stress indices that induce atherosclerosis: mean (over the cardiac cycle), maximum and minimum, pulse (maximum - minimum), negative index, NEG (fractional time during which the shear stress stays negative) and oscillatory shear stress index (OSI). Reneman et al. (1993) used experimental and computational models to study flow in bifurcation regions. Gijsen et al. (1997) suggested a new experimental technique to determine the wall shear stress in vivo. Vorp et al. (1998) used a coupled fluid structure interaction model to combine the influence of mechanical stress and wall shear stress and concluded that the arterial diseases are most probably localized in regions of high mechanical stress and low wall shear stress. However, the mechanical stress within the wall cannot easily be extracted unless further development in imaging techniques and image segmentation algorithms are achieved. In this study, we use

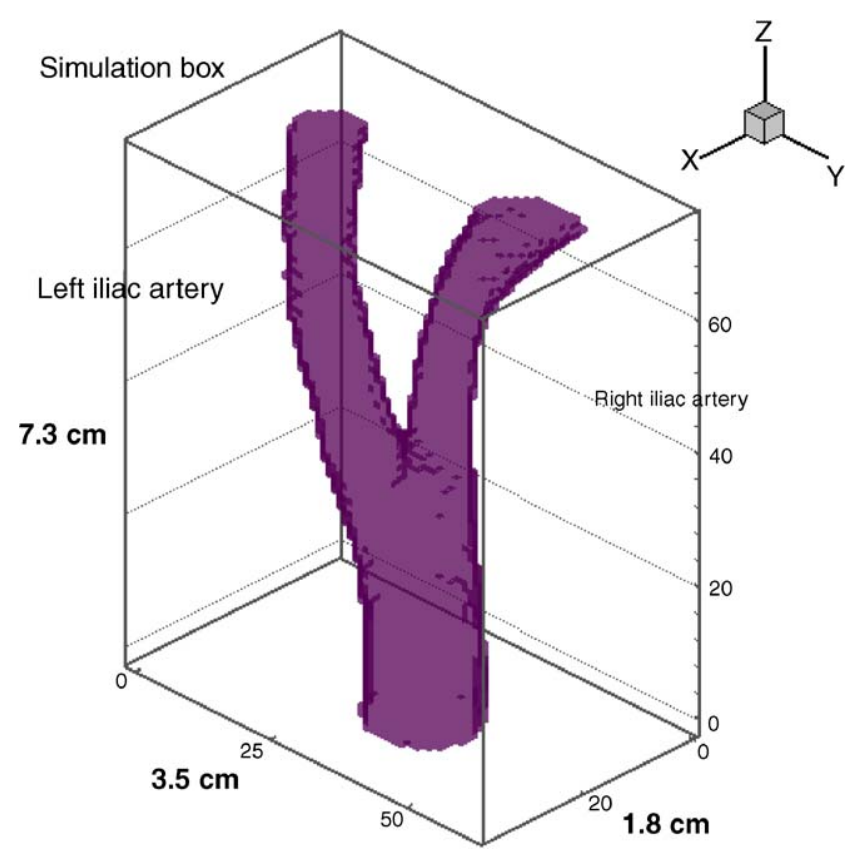

Fig. 2. An MRA reconstructed model of the aortic bifurcation with left and right iliacs. The right iliac is more bended than the left one. The computational grid size is 164,761 nodes.
Eq. (3) to compute the nine components of the symmetric stress tensor, i.e. $\sigma_{\alpha \beta}$ which represents the stress component in the $\beta$-direction acting on the element with outward normal in the $\alpha$-direction. Since the components of any second-order tensor can be reduced to an eigenvalue problem, it is possible to transform the second-order stress tensor into an eigenvalue problem and extract the principal stresses $\lambda_{1}, \lambda_{2}$ and $\lambda_{3}$ with their eigenvectors. These can be visualized as a quadric surface (elliptical glyphs), but in biomechanics, the traction forces, shear stress and the von Mises effective stress are commonly used. The traction forces are orientation dependent and need the surface normals. However, the von Mises effective stress, usually available in visualization packages, is computed from the second invariant of the stress tensor (see the next section) and is more suitable for Cartesian grids than the surface tractions, as the Cartesian grids introduce additional approximations when computing surface normals. The von Mises stress is commonly used in biomechanics to determine the effective stress (e.g.. Raghavan and Vorp, 2000). In this study, we will focus on the time-behavior of the effective von Mises stress.

For our simulations, a smoothed MRA image was provided by Charles Taylor, Stanford University, USA, with original resolution of $512 \times 512 \times 64$ voxels, each voxel occupies 1 byte. The spacing between each two successive recorded cut planes is $0.9375 \mathrm{~mm}$. An image segmentation algorithm is applied to the original data set to extract the aorta and the segmented aorta is then cropped and filtered to end up with the simulation model shown in Fig. 2.

It is worth noting that there is a limitation on obtaining high-resolution images for the arterial system. To have a stable solution in the lattice Boltzmann method, the relaxation time $\tau$ must be larger than 0.5 in order to have a positive fluid viscosity. Having a Reynolds number $R_{\mathrm{e}}$ within a diameter $n D, n$ being the spatial resolution of the image, for a fluid of blood viscosity, will result in a relaxation time of $0.5+$ $0.3 n D / R_{\mathrm{e}}$. Therefore, the stability scales linearly with the spatial resolution.

We have conducted a number of steady and unsteady flow simulations for the aorta model. As the cross section of each slice is irregular, the Reynolds number is redefined as $\operatorname{Re}=4 m U / v=4 Q / v P$ and the Womersley number is defined as $\alpha=2 m \sqrt{\omega / v}$, where $m$ is the mean hydraulic depth which is the ratio between the vascular bed $A$ and the perimeter $P$. Fig. 3 shows changes in the Reynolds and the Womersley numbers downstream of the aortic model under resting and exercise conditions, assuming a flow rate of $0.81 / \mathrm{min}$ with 65 beats $/ \mathrm{min}$ under resting and $5.361 / \mathrm{min}$ with $130 \mathrm{beats} / \mathrm{min}$ for exercise conditions (Moore and Ku, 1994b).

The steady flow simulations are performed to assess the steady flow behavior and to check the validity of the 

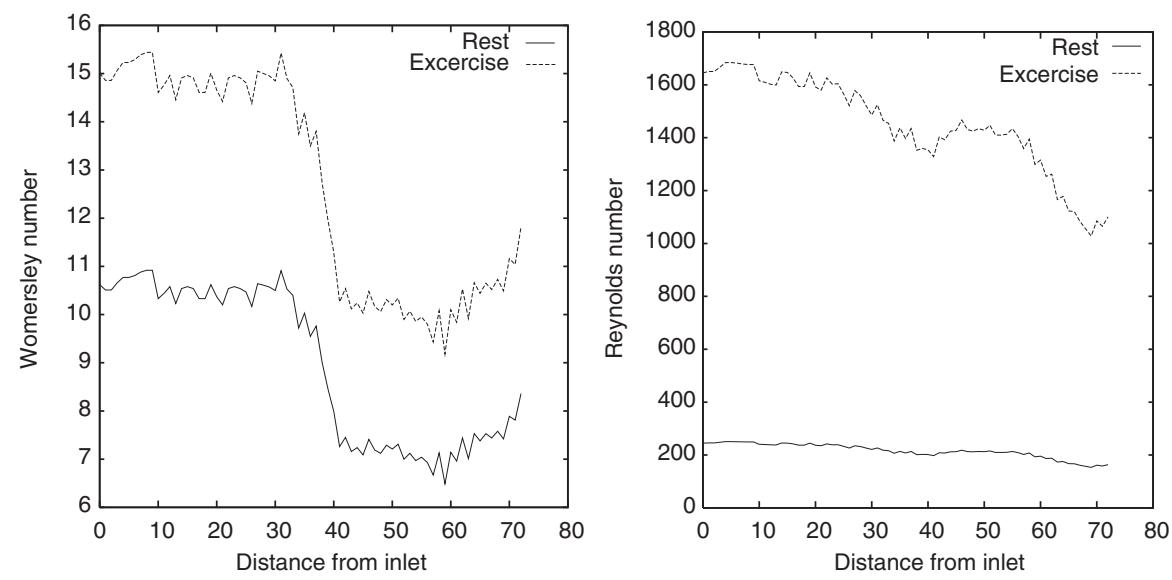

Fig. 3. Change in Womersley parameter (left) and Reynolds number (right) along the segmented aorta during resting and exercise conditions.

used inlet and outlet conditions. We have used the bounce-back rule as a wall boundary condition. For the inlet, we use an assigned inlet pressure to compute the inlet velocity (Zou and $\mathrm{He}, 1997$ ) and assign equilibrium values for unknown distributions. The outlet conditions are assigned accordingly with an outlet pressure. The maximum Reynolds number is 1500 . A velocity snapshot of steady flow is shown in Fig. 4, from which we observe that the bended branch of the aorta (the right iliac in this case) has less entrance velocity than the less curved one (the left iliac). The velocity gradients before the bifurcation are smaller near to the right lateral wall than those on the left lateral wall and the shear stress is expected to be smaller. Also, the posterior wall receives less velocity than the anterior wall and a similar conclusion may be drawn. However, the unsteady nature of the locality of low shear stress may be different as will be explained later.

For the unsteady flow simulations, an aortic pressure waveform is applied at the entrance of the aortic model. Velocities are then computed from the distribution functions coming from downstream, and the unknown distributions are set to their equilibriums. At the two outlets, constant pressure is applied. The total simulation time is $2 \mathrm{~h}$ on a single processor and reduces to 40 min when using 4 nodes. We assume that the system has converged after the change in conserved quantities (mass and momentum) is less than $2 \times 10^{-5} \%$. This results in an error that is less than $1 \%$ when simulating rigid circular tubes, as claimed in the previous section. At least 40 complete periods are needed to converge to the simulation criterion. Although it seems longer than the required periods when using a finite element solver, the total simulation time per period is far less (Taylor et al., 1998). On a single processor, a period represented by 240 time-steps takes approximately $3 \mathrm{~min}$. Flow fields and shear stress are recorded during the last cycle as separate frames for each time-step. The phase of the full cardiac cycle $\left(360^{\circ}\right)$ is split into a number of frames

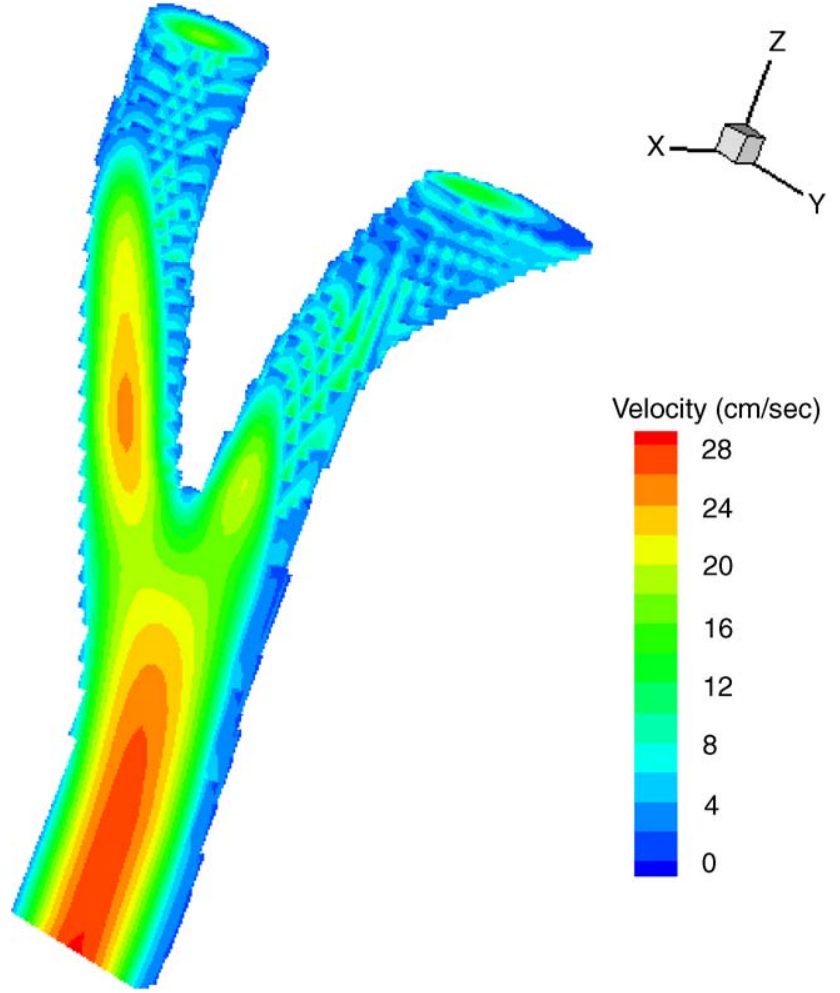

Fig. 4. Steady flow in the aortic bifurcation. The maximum Reynolds number is 1500 .

(vertical lines in Fig. 5) and each frame is named after the corresponding phase angle. Vector magnitudes of velocity profiles at 10 frames are visualized in Fig. 5.

At the beginning of systole (frame 0 ), the flow through the bifurcation is relatively simple, except for a very small velocity of maximum magnitude $5.0 \mathrm{~cm} / \mathrm{s}$ near to the walls of the main branch (see Fig. 6). As the flow is increased (frames 36 and 72), the velocity increases rapidly in the main branch and slowly in the iliacs. The left iliac receives more flow than the right one (see the change in the red dot on top of the branch). 

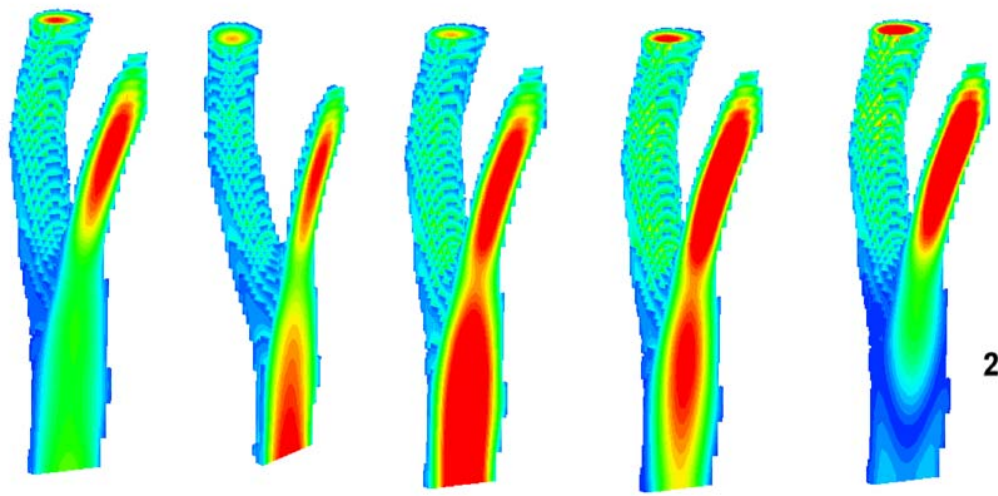

$20 \mathrm{~cm} / \mathrm{s}$

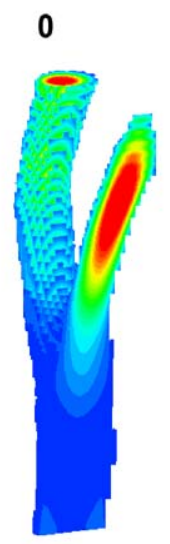

36

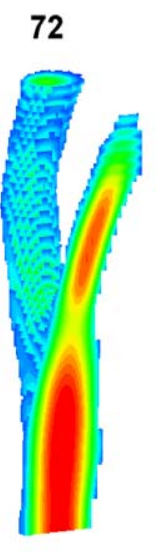

108

144
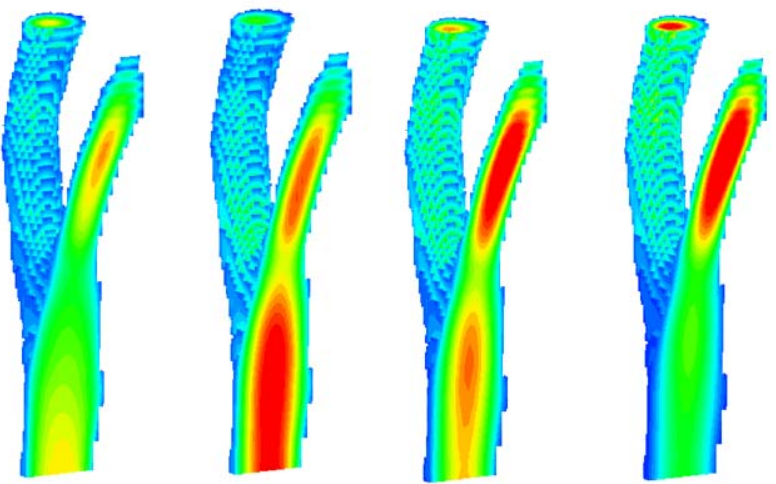

288

324

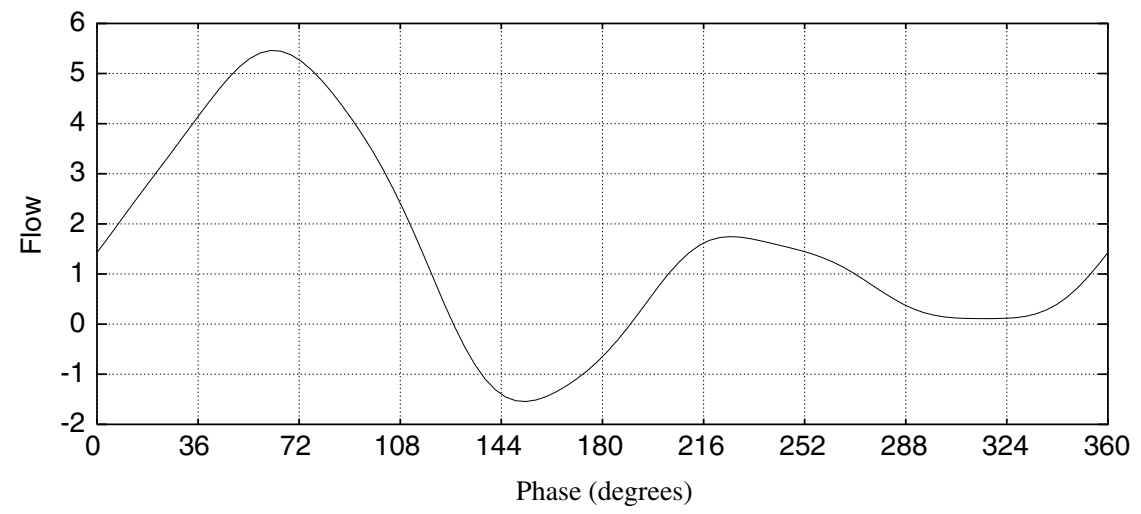

Fig. 5. Velocity profiles in the aortic bifurcation computed at every $36^{\circ}$ of the cardiac cycle, at 120 heart beats $/$ min and a flow rate of $90 \mathrm{~cm}^{3} / \mathrm{s}$.

The flow then relaxes towards the end of systole (frame 108). After that, it becomes more complex, especially in the main branch (see frames 144 and 180), involving two conjugate vortices and flow mixing. This is clearly illustrated by streamlines shown in Fig. 7. It is worth noting that, although the flow reverses in the main branch during this period, the flow at the exits is forward on average. This demonstrates the function of the aorta as a reservoir that provides blood to the organs when the flow reverses. The second half of the cardiac cycle represents the diastole (frames 216-324) during which the flow oscillates till it reaches the beginning of systole where frame 0 is repeated.

Velocity magnitudes near the posterior wall are approximately the same as those close to the anterior wall, as shown by the symmetry in color. Throughout most of the cardiac cycle, the flow is slightly skewed toward the anterior wall (Fig. 5).

Close to the bifurcation, the flow becomes quite complex. At about $15 \mathrm{~mm}$ proximal to the bifurcation, the flow reverses near the walls during most of the cardiac cycle. It was reported that the walls proximal to 


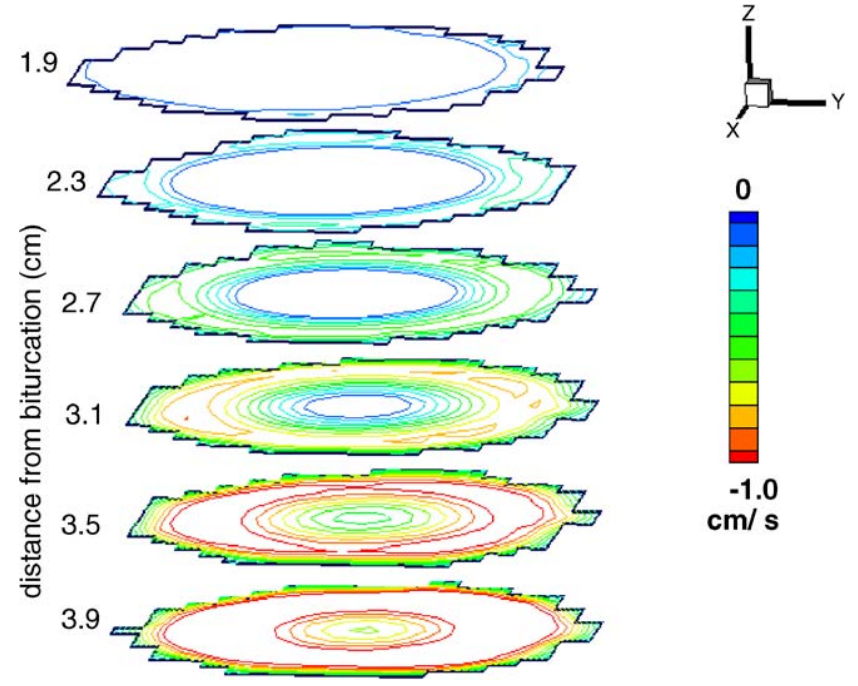

Fig. 6. Negative velocity profiles during the systole are frequently observed close to the aortic bifurcation. The figure shows two snapshots of the velocity, $2.0 \mathrm{~cm}$ proximal to the bifurcation.
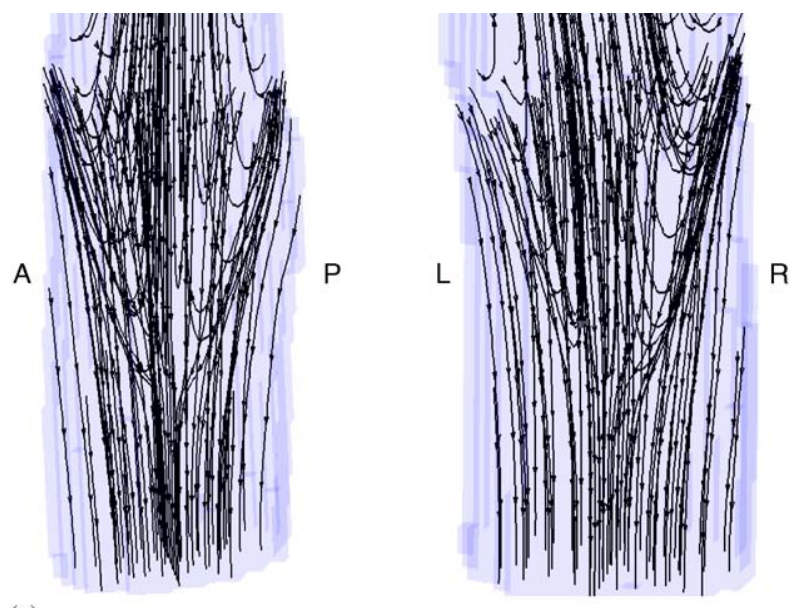

(a)
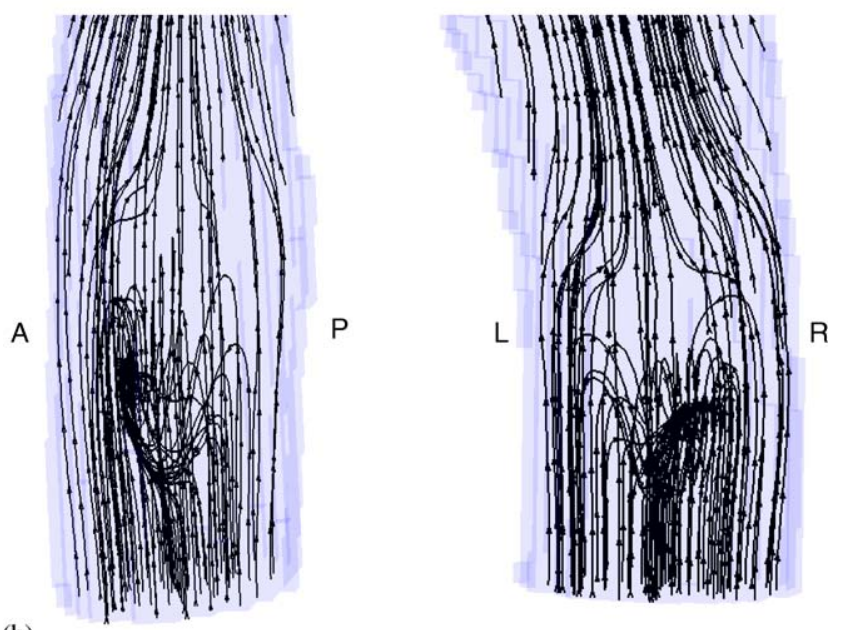

(b)

Fig. 7. Velocity streamlines showing: (a) vortex formation during the diastole (at $t=0.4 \mathrm{~T}$ ) and (b) flow mixing (at $t=0.5 \mathrm{~T}$ ). the aorta are frequently subjected to occlusive atherosclerosis, although this region does not involve bifurcation or area expansion which are two major factors that complicate the flow pattern (Moore and $\mathrm{Ku}$, 1994a). The locality of atherosclerosis in this straight segment is attributed to the low and oscillatory near wall velocity profiles, which may result in mass transfer from blood to the walls (Moore and Ku, 1994a; Taylor et al., 1996).

After the bifurcation, the flow is laminar. The left iliac receives more flow during the systole. In order to have a clearer picture about the flow, streamlines are plotted for the whole cardiac cycle. Except for periods of back flow, the streamlines are in general uniform and show forward direction of the flow (data not shown). Vortex rings set up during flow reversal at the end of systole (see Fig. 7), but they progressively damp out when the flow is re-established. These vortices form a trap for fluid elements and disturb the flow across the whole vessel. The reason for formation of vortices may be attributed to the rapid flow reversal and the damp-out may be forced by the inherent stability of the flow. Some of these observations have been previously reported by Moore and $\mathrm{Ku}$ (1994a) in their extensive experimental MRA velocity measurements of a glass blown idealized model of the abdominal aorta and by Taylor et al. (1996) who observed large vortex development along the wall of the abdominal aorta. They claimed that this vortex shrinks considerably under moderate exercise condition. It is worth noting that although the models investigated by Moore and Ku (1994a) and by Taylor et al. (1996) are idealized, similar qualitative results could be observed.

\subsection{Shear stress}

Shear stress for a Newtonian fluid is conventionally estimated from gradients of measured or simulated velocity components, and the fluid viscosity. This process involves some approximations which may lead to underestimation on the order of $10-45 \%$ (Luo et al., 1993) when the lumen is not circular. This large error is due to the nonlinear velocity profile at the wall and ignorance of the radial derivatives. Enhancement was recently reported by Cheng et al. (2002) by introducing piecewise Lagrangian basis functions and segmenting the vessel lumen with a level set method.

With the LBM, the nine Cartesian components of the local stress tensor are directly obtained using Eq. (3), as stated above without any further approximation than the Cartesian geometry. The stress at any given point is completely determined by this stress tensor. A real benefit of the lattice Boltzmann solver is that these components are computed independent from velocity gradients. In this study, we compute and visualize the von Mises effective stress (Geiringer, 

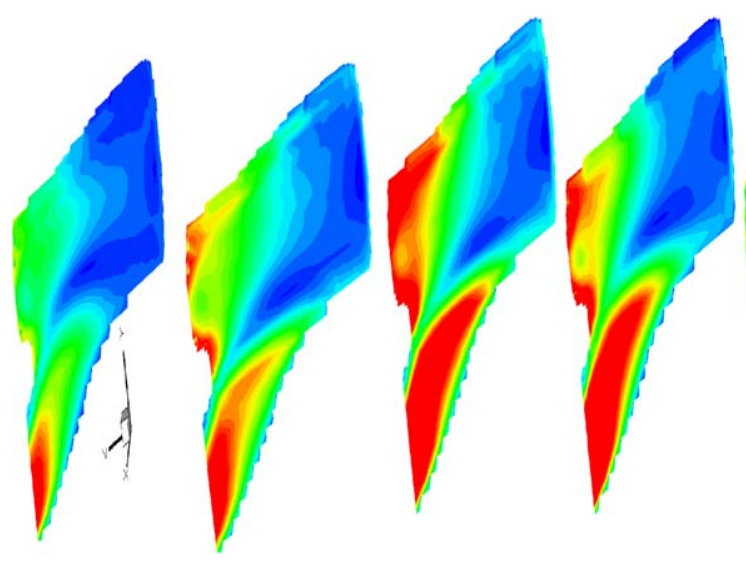

36

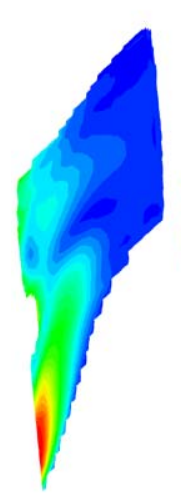

180

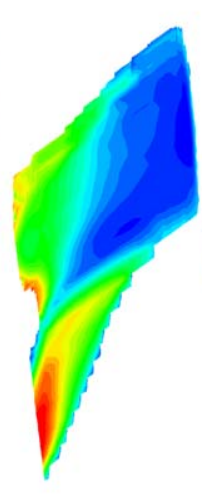

216

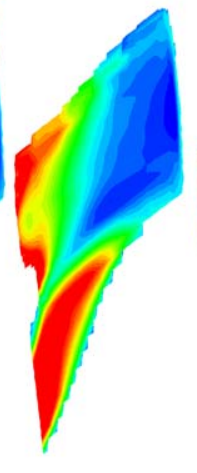

252

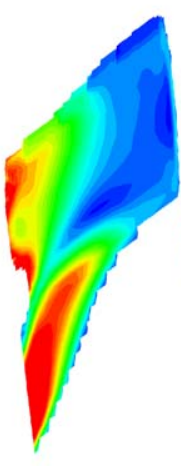

288

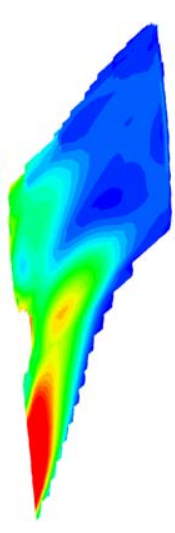

144

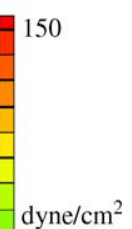

72

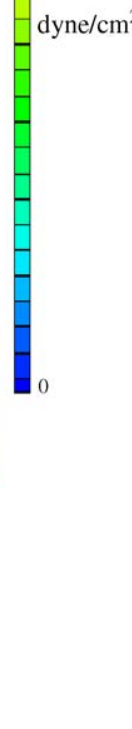

354

Fig. 8. Effective stress on the walls of the aortic bifurcation computed at every $36^{\circ}$ of the cardiac cycle. The posterior wall has low values throughout the whole systolic cycle, while relatively high values of the stress near the curved exits are observed.

1953), defined as

$\sigma_{\text {eff }}=\sqrt{\frac{A+6 B}{2}}$

where

$A=\left(\sigma_{x x}-\sigma_{y y}\right)^{2}+\left(\sigma_{y y}-\sigma_{z z}\right)^{2}+\left(\sigma_{z z}-\sigma_{x x}\right)^{2}$

and

$B=\sigma_{x y}^{2}+\sigma_{y z}^{2}+\sigma_{z x}^{2}$.

This quantity is one of the three invariants of the stress tensor and therefore, is orientation independent. In addition, it includes the effect of small directional variations in the octahedral normal stress (the mean pressure) on the walls. The quantity $B$ vanishes in the principal coordinate system.

The effective stress in dynes $/ \mathrm{cm}^{2}$ is shown in Fig. 8, from which we observe that the effective stress at the posterior and lateral walls is always small (less than
40 dynes $/ \mathrm{cm}^{2}$ ) throughout the cardiac cycle. The stress is uniformly distributed along the lateral walls. The minimum stress values are observed during the flow reversal where the stress is very small through the whole vessel. The stress is also oscillating in magnitude and the stress vector oscillates rapidly. The high stress values near the exits of the left iliac are attributed to the fact that this branch is subject to a small curvature at these locations. Effects of outlet conditions are minor and are hardly seen. The stress behavior during the systole is described below. Let us first describe the stress behavior in the main branch. At the beginning of systole (frame 0 ), the shear stress is small (less than 20 dynes $/ \mathrm{cm}^{2}$ ) and is least around the walls, with the posterior and right lateral walls having minimum values close to zero. The lateral walls of the left iliac have higher shear stress than the lateral walls for the right iliac, as predicted by the steady flow simulations. The shear stress increases close to the walls as the systolic pressure is increased (frame 
36) a strip-like island of zero shear stress splits the region just before the bifurcation into a left and right regions, with the left region having higher stress than the right one. The left anterior walls receive more stress (frame 72) of magnitude greater than 60 dynes $/ \mathrm{cm}^{2}$. Then the walls around the main are released from regions of minimum stress (frame 108), with the posterior walls released first while the anterior walls are not (frame 144). When the systole ends and the diastole begins (frame 180), the shear stress becomes again very small at regions far from the bifurcation, with small islands in the center with minimum shear stress. The stress on the anterior lateral walls then increases, except for a small island in the middle of posterior wall, just before the bifurcation (frame 216). The shear stress comes again to its minimum on the centers and at the distal posterior walls (frame 252), with maximum shear stress $\left(40\right.$ dynes $\left./ \mathrm{cm}^{2}\right)$ on the walls. Then it gets smaller again, except near the left anterior walls (frame 288). Near the end of the cardiac cycle (frame 324), the shear stress is low around the walls and on islands close to the bifurcation, spreading from left posterior to right anterior and covering the right anterior sides. The left anterior walls has clearly larger shear stress than the right side. At the end of the cycle, frame 0 is repeated. In summary, the posterior wall receives stresses greater than 60 dynes $/ \mathrm{cm}^{2}$ during onethird of the cycle, less than 5 dynes $/ \mathrm{cm}^{2}$ during another third and between 20 and 40 dynes $/ \mathrm{cm}^{2}$ during the rest of the cycle. The shear stress after the bifurcation is higher in magnitude than the main branch, except for some islands and edges.

At the beginning of systole (frame 0), the regions directly after the bifurcation have minimum shear stress in an island on the right iliac spreading toward the right lateral and posterior walls, while the left iliac has higher magnitudes on the outer walls and minimum values on the inner walls. The bend near the exit makes the shear stress highest at these locations $\left(>150\right.$ dynes $\left./ \mathrm{cm}^{2}\right)$. The stress is also maximum in the center of the exit of left iliac artery and one-third from the exit of the right iliac. The inner walls of the right iliac receives minimum shear stress. The near-end (after the bend) outer walls receive minimum shear stress. In summary, at the beginning of systole, the inner walls have less stress than the outer ones and the right iliac artery has less shear stress than the left one.

As the systole develops, the stress first goes higher towards the bifurcation (frame 36), but remains minimum for some islands on the right iliac: close to the bifurcation and near the inner posterior walls (frame 72). Near the exits, the stress at the anterior walls of the left iliac becomes minimum at the bend (frame 108), and gets less for the inner walls. The islands in the right iliac are shifted towards the posterior inner walls (frames 144 and 180) till they are accompanied by high stress islands spreading toward the outer and anterior walls of both iliacs (frame 216). The stress reaches its maximum directly after the beginning of diastole (frame 252) and oscillates around lesser values (frames 288 and 324) till the systole begins again (frame 0 ).

From this description, we see that the effective von Mises stress is minimum close to the lateral and posterior walls of the abdominal aorta segment before the bifurcation, at the inner walls of the iliacs, and at islands in the right iliac artery, and is maximum at anterior walls, outer walls of the iliacs and at islands on both iliacs. Comparable results have been obtained in the literature (e.g. Raghavan and Vorp, 2000; Moore et al., 1994b; Taylor et al., 1996) leading to similar conclusions about the relationship between localization of cardiovascular diseases and the complex nature of stress. In this study, we did not measure the oscillatory shear index, although it is known to have an influence on the locality of cardiovascular diseases. This will be addressed in future work where the full abdominal aorta will be studied.

\section{Summary}

We have demonstrated that the lattice Boltzmann method is a successful mesoscopic solver to timedependent blood flow in an arterial system. Simulated results of systolic flow in a 3D rigid tube at hemodynamic Reynolds and Womersley parameters have recovered the analytic Womersley solutions within acceptable accuracy. Steady and unsteady flow fields in a realistic aorta geometry, reconstructed from magnetic resonance angiography have been successfully obtained and compared to the available literature, showing qualitative agreements. As the shear stress plays a crucial role in cardiovascular diseases and since it is directly and independently computed in the lattice Boltzmann solver, we strongly encourage researchers from hemodynamics to consider this method as an alternative blood flow solver. More benefits are seen from easy grid generation and straightforward parallelism, easy and feasible adaptation to changing geometry. Further investigation of the complete aorta model and experimental validations are under development in our group.

\section{Acknowledgements}

We thank Prof. Charles Taylor, Stanford University for providing the segmented aorta data set and Robert Belleman for Cartesian grid generation of the model under study. 


\section{References}

Aharonov, E., Rothman, D.H., 1993. Non-Newtonian flow (through porous-media) - a lattice-Boltzmann method. Geophysical Research Letters 30, 679-682.

Artoli, A.M., Hoekstra, A.G., Sloot, P.M.A., 2002. 3D pulsatile flow with the lattice Boltzmann BGK method. International Journal of Modern Physics C13, 1119-1134.

Batina, T., 1993. A gridless Euler/Navier-Stokes solution algorithm for complex aircraft applications. In: Proceedings of the 31st Aerospace Sciences Meeting and Exhibit, American Institute of Aeronautics and Astronautics, Reno, NV.

Behrend, O., 1995. Solid-fluid boundaries in particle suspension simulations via the lattice Boltzmann method. Physical Review E 52 (1), 1164-1175.

Belleman, R.G., Sloot, P.M.A., 2000. The design of dynamic exploration environments for computational steering simulations. In: Proceedings of the SGI Users Conference, Academic Computer Centre CYFRONET AGH, Krakow, Poland.

Berthier, B., Bouzerar, R., Legallais, C., 2002. Blood flow patterns in an anatomically realistic coronary vessel: influence of three different reconstruction methods. Journal of Biomechanics 35, $1347-1356$

Botnar, R., Rappitsch, G., Scheidegger, M.B., Liepsch, D., Perktold, K., Boesiger, P., 2000. Hemodynamics in the carotid artery bifurcation: a comparison between numerical simulations and in vitro MRI measurements. Journal of Biomechanics 33, 137-144.

Caro, C.G., Pedley, T.J., Schroter, R.C., Seed, W.A., 1978. The Mechanics of the Circulation. Oxford University Press, Oxford.

Cheng, C.P., Parker, D., Taylor, C.A., 2002. Quantification of wall shear stress in large blood vessels using lagrangian interpolation functions with cine phase-contrast magnetic resonance imaging. Annals of Biomedical Engineering 30, 1020-1032.

Chopard, B., Droz, M., 1998. Cellular Automata Modeling of Physical Systems. Cambridge University Press, Cambridge.

Filippova, O., Hänel, D., 1998. Boundary-fitting and local grid refinement for lattice-Bgk models. International Journal of Modern Physics C 9 (8), 1271-1279.

Geiringer, H., 1953. Recent results in the theory of plasticity. In: von Mises, R., von Karmen (Eds.), Advances in Applied Mechanics III. Academic Press, New York, pp. 200-201.

Gibson, C.M., Diaz L., Kandarpa, k., Sacks, F.M., Pastenak, R.C., Sandoor, T., Feldman, C., Stone, P.H., 1993. Relation of vessel wall shear-stress to Atherosclerosis progression in human coronary-arteries. Arteriosclerosis and Thrombosis 13(2), 310-315.

Gijsen, F.J.H., Goijaerts, A.M., van de Vosse, F.N., Janssen, J.D., 1997. A new method to determine wall shear stress distribution. Journal of Rheology 41 (5), 995-1006.

Goyen, M., Ladd, M.E., Debatin, J.F., Barkhausen, J., Truemmler, K.H., Bosk, S., Ruehm, S.G., 2001. Dynamic 3D MR angiography of the pulmonary arteries in under four seconds. Journal of Magnetic Resonance Imaging 13(3), 372-377.

Higuera, F.G., Succi, S., 1989. Simulating the flow around a circularcylinder with a lattice Boltzmann-equation. Europhysics Letters 8 (6), 517-521.

Hoekstra, A.G., van't Hoff, J., Artoli, A.M., Sloot, P.M.A., 2004. Unsteady flow in a 2D elastic tube with the LBGK method. Future Generation Computer Systems 20, 917-924.

Kandhai, D., Vidal, D.J., Hoekstra, A.G., Hoefsloot, H., Iedema, P., Sloot, P.M.A., 1999. Lattice-Boltzmann and finite element simulations of fluid flow in a SMRX static mixer reactor. International Journal of Numerical Methods in Fluids 31, 1019-1033.

Krafczyk, M., Cerrolaza, M., Schulz, M., Rank, E., 1998. Analysis of 3D transient blood flow passing through an artificial aortic valve by Lattice-Boltzmann methods. Journal of Biomechanics 31, 453-462.
Ku, D.N., Giddens, D.P., Zarins, C.K., Glagov, S., 1985. Pulsatile flow and atherosclerosis in the human carotid bifurcation: positive correlation between plaque location and low and oscillating shear stress. Arteriosclerosis 5, 293-302.

Ladd, A.J.C., 1994. Numerical simulations of particulate suspensions via a discretized Boltzmann equation. Part I. Theoretical foundation. Journal of Fluid Mechanics 271, 285-309.

Luo, Z., Yang, W.J., Stein, P.D., 1993. Errors in the estimate of arterial wall shear rated that result from curve fitting of velocity profiles. Journal of Biomechanical Engineering 26, 383-390.

McDonald, D.A., 1974. Blood Flow in Arteries. Camelot Press.

Mcnamara, G.R., Zanetti, G., 1988. Use of the Boltzmann-equation to simulate lattice-gas automata. Physical Review Letters 61 (20), 2332-2335.

Moore, J.E., Ku, D.N., 1994a. Pulsatile velocity measurements in a model of the human abdominal aorta under resting conditions. Journal of Biomedical Engineering 116, 337-346.

Moore, J.E., Ku, D.N., 1994b. Pulsatile velocity measurements in a model of the human abdominal aorta under resting simulated exercise and postprandial conditions. Journal of Biomedical Engineering 116, 107-116.

Moore, J.E., Maier, S.E., Ku, D.N., Boesiger, P., 1994a. Hemodynamics in the abdominal aorta: a comparison of in vitro and in vivo measurements. Journal of Applied Physiology 76, 1520-1527.

Moore, J.E., Xu, C., Glagov, S., Zarins, C.K., Ku, D.N., 1994b. Fluid wall shear stress measurements in a model of the human abdominal aorta: oscillatory behavior and relationship to atherosclerosis. Atherosclerosis 110, 225-240.

Moore, J.A., Steinman, D.A., Ethier, C.R., 1998. Computational blood flow modelling: errors associated with reconstructing finite element models from magnetic resonance images. Journal of Biomechanics 31, 179-184.

Oshinski, J.N., Ku, D.N., Mukundan, S., Loth, F., Pettigrew, R.I., 1995. Determination of wall shear stress in the aorta with the use of MR phase velocity mapping. Journal of Magnetic Resonance Imaging 5(6), 640-647.

Qian, Y.H., DHumieres, D., Lallemand, P., 1992. Lattice BGK models for Navier-Stokes equations. Europhysics Letters 17, 479-484.

Raghavan, M.L., Vorp, D.A., 2000. Toward a biomechanical tool to evaluate rupture potential of abdominal aortic aneurysm: identification of a finite strain constitutive model and evaluation of its applicability. Journal of Biomechanics 33, 475-482.

Reneman, R.S., Hoeks, A.P.G., van de Vosse, F.N., Ku, D.N., 1993. Three-dimensional blood flow in bifurcations: computational and experimental analyses and clinical applications. Cerebrovascular Diseases X, 185-192.

Shaaban, A.M., Duerinckx, A.J., 2000. Wall shear stress and early atherosclerosis: a review. American Journal of Roentgenology 174, $1657-1665$.

Succi, S., 2001. The Lattice Boltzmann Equation for Fluid Dynamics and Beyond. Oxford University Press, Oxford.

Taylor, C.A., Hughes, T.J.R., Zarins, C.K., 1996. Computational investigations in vascular disease. Computers in Physics 10(3), 224-232.

Taylor, C.A., Hughes, T.J.R., Zarins, C.K., 1998. Finite element modeling of blood flow in arteries. Computer Methods in Applied Mechanics and Engineering 158, 155-196.

Taylor, C.A., Darney, M.T., Ku, J.P., Parker, D., Steele, B.N., Wang, K., Zarins, C.K., 1999. Predictive medicine: computational techniques in therapeutic decision making. Computer Aided Surgery 4, 231-247.

Vorp, D.A., Raghavan, M.L., Webster, M.W., 1998. Mechanical wall stress in abdominal aortic aneurysm: influence of diameter and asymmetry. Journal of Vascular Surgery 27 (4), 632-639. 
Womersley, J.R., 1955. Method for calculation of velocity, rate of flow and viscous drag in arteries when the pressure gradient is known. Journal of Physiology 127, 553-563.

Wootton, D., Ku, D.N., 1999. Fluid mechanics of vascular systems diseases and thrombosis. Annual Review of Biomedical Engineering 1, 299-329.

World Health Organization, 2002. The World Health Report 2002 Reducing risks, promoting healthy life, World Health Organization, http://www.who.int/whr/en/.
Zhao, Z., Belleman, R.G., van Albada, G.D., Sloot, P.M.A., 2002. AG-IVE: an agent based solution to constructing interactive simulation systems. In: Computational ScienceICCS 2002, Proceedings Part I, in series Lecture Notes in Computer Science, vol. 2329. Springer, Berlin, pp. 693-703.

Zou, Q., He, X., 1997. On pressure and velocity boundary conditions for the lattice Boltzmann BGK model. Physics of Fluids 9(6) 1591-1598. 